\title{
EL APEGO EN LA VEJEZ, UNA DIMENSIÓN A TENER EN GUENTA
}

\author{
ATTACHMENT IN OLD AGE, \\ A DIMENSION TO TAKE INTO AGGOUNT
}

\author{
M. PILAR QUIROGA-MÉNDEZ ${ }^{1}$
}

Cómo referenciar este artículo/How to reference this article:

Quiroga-Méndez, M. P. (2020). El apego en la vejez, una dimensión a tener en cuenta [Attachment in Old Age, a Dimension to Take into Account]. Acción Psicológica, 17(2), 13-24. https://doi.org/10.5944/ap.17.2.29838

\section{Resumen}

La teoría del apego aporta un marco nuevo para la comprensión de la vejez. Por sus características de respuesta de activacion automática ante cualquier situación percibida como amenazante, el apego es un constructo especialmente importante en la vejez. Se postula el apego como el factor precursor de la regulación emocional del anciano, por su automatismo, estabilidad en el ciclo vital y conexión con mecanismos biológicos básicos de la ansiedad. Los apegos resistentes actúan en la vejez produciendo respuestas de extrema dependencia y sufrimiento; y los tipos evitativos activan una independencia excesiva, que impide el autocuidado y la percepción de riesgo. Ademas los problemas de salud propios de la vejez, se agravan en los sujetos con apego inseguro, creando una mayor vulnerabilidad a la enfermedad por los procesos biológicos de tipo inflamatorio y cardiaco, subyacentes a este tipo de apegos. Más de la mitad de la población mayor puede considerarse población de riesgo, debido a la presencia de apegos inseguros. A través de la teoria del apego se pueden comprender las reacciones de las personas mayores sometidas a tensión e intervenir de un modo ajustado, pues esta perspectiva se convierte en moduladora y novedosa para la comprensión del envejecimiento patológico y saludable.

Palabras clave: teoría del apego; vejez; enfermedad; regulación emocional.

\begin{abstract}
Attachment theory provides a new framework for understanding old age. Due to its automatic activation response characteristics to any situation perceived as threatening, attachment in old age is an especially important construct. Attachment is postulated as the precursor factor of emotional regulation in the elderly, due to its automatism, sta-
\end{abstract}

Correspondence address [Dirección para correspondencia]: María Pilar Quiroga Méndez, Facultad de Psicología, Universidad Pontificia de Salamanca, España.

Email: quiroga@upsa.es

ORCID: María Pilar Quiroga Méndez (https://orcid.org/0000-0001-6408-8855)

${ }^{1}$ Facultad de Psicología. Departamento de Psicologia Evolutiva, Universidad Pontificia de Salamanca.

Recibido: 1 de marzo de 2020.

Aceptado: 21 de noviembre de 2020. 
bility in the life cycle and connection with basic biological mechanisms of anxiety. Resistant attachments act in old age, producing responses of extreme dependence and suffering; and avoidance types activate excessive independence, which prevents self-care and the perception of risk. In addition, health problems typical of old age are aggravated in insecurely attached subjects, creating a greater vulnerability to disease due to the inflammatory and cardiac biological processes underlying this type of attachment. More than half of the elderly population can be considered a population at risk, due to the presence of insecure attachments. Through attachment theory, it is possible to understand the reactions of older people under stress and intervene in a tight way, since this perspective becomes modulating and novel for the understanding of old age.

Keywords: attachment theory; old age; disease; emotional regulation.

\section{Introducción}

La teoría del apego ha dado lugar a investigación e intervenciones clínicas durante más de 50 años, en los que se han asociado los patrones de apego con un amplio rango de síntomas y patologías, así como con la capacidad de regulación emocional, bienestar, salud, resiliencia e incremento de la vulnerabilidad. Estos aspectos son especialmente importantes en la vejez, y por ello resulta incomprensible que el apego aplicado a esta época de la vida, siga teniendo una incomparable menor atención que el apego infantil o el apego adulto. Apenas un $0.52 \%$ del total de publicaciones sobre apego, se refieren al apego en los mayores (Quiroga, 2018). En la vejez la enfermedad, y la percepción de fragilidad aumentan, por tanto, las respuestas de apego también se acrecientan, ocasionando dificultades emocionales y de salud que requieren de esta perspectiva teórica para su comprensión. Este trabajo pretende actualizar un constructo que ha demostrado su utilidad en todas las etapas de la vida, y sin embargo permanece ausente en la época de la vejez, exponiendo su valor heurístico y ampliando un campo imprescindible para la propia teoría del apego y sobre todo para la comprensión de la vejez.

\section{Apego y enfermedad. Los apegos inseguros como factores de riesgo de enfermedad en la vejez}

Sobre la vivencia de los primeros vínculos, y experiencias del yo con su entorno de cuidado, las personas desarrollan un guion, sistema o prototipo denominado «sistema de comportamiento del apego» o Working Model (WM), que funcionará como un esquema aplicable a las relaciones que implican vínculos emocionales, demostrando estabilidad y relativa continuidad a lo largo del ciclo vital. La investigación porterior ha añadido a este esquema inicial cognitivo los procesos biológicos involucrados en la respuesta de apego. Estos trabajos comienzan con las investigaciones de Hofer (2006), que identifican subsistemas psicofisiológicos ocultos, que se interrumpen cuando las crias son retiradas de sus cuidadores, tales como los que controlan la frecuencia cardíaca, la temperatura corporal, la ingesta de alimentos y la exploración (McMahon et al., 2019). En investigación animal también se ha encontrado la permanencia de estos efectos durante la edad adulta (Meany, 2001) comprobando incluso la influencia en la expresión génetica de los descendientes, un hallazgo que ha abierto nuevas áreas de investigación (Ein Dor et al., 2018; Iyengar, 2019).

La neurociencia del apego investiga los correlatos neuronales identificando las estructuras cerebrales, neurotrasmisores y neuropéptidos que proporcionan las evidencias de que los distintos tipos de apego tienen su correspondiente neurológico (Coan, 2008; Vrtička y Vuilleumier, 2012). Existe una creciente evidencia de que el eje HPA, un sistema de respuesta al estrés, completamente desarrollado y presente al nacer, sufre variaciones en su calibración original y regulación contínua, en respuesta a las primeras experiencias con los cuidadores (Chen et al., 2011; Pietromonaco et al., 2013). Este sistema, es esencial para la regulación de la respuesta al estrés, y también está en la base de las respuestas inflamatorias, y de reactividad del sistema inmune. En la edad adulta, los sistemas de apego continúan operativos, y los estudios muestran la relación de los diferentes tipos de apego con biomarcadores de inmunidad. Los apegos inseguros evitativos se relacionan con la presencia de niveles elevados de citocina proinfla- 
matoria, interleucina-6 (IL-6) en respuesta a los estresores interpersonales, y niveles más bajos de células NK (Gouin et al., 2009; Picardi et al., 2007). En los apegos inseguros ansiosos, se destaca una producción elevada de cortisol y un menor número de células $\mathrm{T}$, pecursoras de las citocinas (Jaremka et al., 2013; Ketay y Beck, 2017; Shirtcliff et al., 2017). La relación entre el apego, el funcionamiento del eje HPA, la inflamación y el sistema inmunitario, es un área de investigación que ayudará a comprender los procesoso biológicos subyacentes al vínculo del apego (Sippel et al., 2017). Por último, las diferencias individuales en el apego se asocian con la anatomía del cerebro y la actividad a lo largo de la vida, y recientemete se han integrado todas estas aportaciones en un modelo neuroanatómico funcional extendido del apego humano (NAMA; Long et al., 2020).

Los sujetos con apegos inseguros, tienden a desarrollar con más facilidad problemas de salud. Parece que las primeras experiencias adversas crean una especie de células con un «fenotipo proinflamatorio» y una desregulación neuroendocrina, que conduce a la inflamación crónica. Esta inflamación, a su vez, está involucrada en una variedad de enfermedades relacionadas con el envejecimiento, incluyendo enfermedades cardiovasculares e autoinmunes, haciendo que el apego inseguro se convierta en un elemento de riesgo, especialmente en la vejez (Chung et al., 2009; Miller et al., 2011).

\section{Apego y Regulación emocional. El apego como condición y origen de la regulación emocional, observado desde la vejez}

La regulación emocional está claramente implicada en el ajuste y en la promoción del bienestar y la salud en los mayores. Por otra parte, el modelo de activación del apego es imprescindible, porque aporta un esquema previo y consistente sobre el que se desarrolla la actividad y la regulación emocional.

Cuando un sujeto se siente amenazado, se activa automáticamente el sistema de apego, que incluye una primera evaluación y seguimiento del evento que nos amenaza, lle- vando al sujeto a la búsqueda de proximidad a las figuras que nos ofrecen protección, soporte emocional o ayuda frente al peligro. En un segundo momento, aparece la evaluación de la figura de apego, de su disponibilidad y de su capacidad de respuesta como figura de referencia, apoyo o ayuda. El resultado de esta evaluación, configura las diferentes tipologías del apego, y se creará en función de la capacidad y presencia del cuidador, y sobre su efectividad en el cuidado. Estas experiencias con las figuras primarias de apego, repetidas en el tiempo, crean un sentido de seguridad disposicional, en lo que se ha denominado circulo de seguridad. En tercer lugar, aparece una evaluación de la utilidad de búsqueda, estimando si esta ha resultado beneficiosa y organizando los comportamientos de vinculación emocional de forma consistente y estable. Este sistema se desarrolla en la infancia, y permanece operativo en condiciones similares durante toda la vida. En función de la experiencia temprana, se constituyen las diferencias individuales en el apego, responsables en último término, de las reacciones de regulación, de seguridad, hiperactivación o dereactivación emocional (Milkunicer y Shaver, 2019). Los diferentes tipos de apego son por tanto, estrategias de regulación emocional que se activarán siempre que existe una percepción subjetiva de amenaza.

Los apegos denominados de tipo inseguro, aparecen porque se han sufrido interrupciones o fallos en los vínculos iniciales, de forma que los sujetos se protejen intentando un ajuste con una figura de apego inconsistente o no disponible. El tipo inseguro-ansioso espera constantemente la existencia de una figura externa que podrá hacerse cargo de la amenaza. Este comportamiento interfiere en el desarrollo de recursos internos, en la búsqueda eficiente de apoyo y en la evaluación objetiva del peligro, ya que, desde la dependencia al cuidador, el sujeto se vive a si mismo como disminuido e ineficiente, y la amenaza es evaluada fácilmente como desmesurada. El apego ansioso tiende a exagerar la gravedad de la amenaza, y atiende preferentemente a los indicadores internos de angustia. La atención se enfoca prioritariamente a los aspectos fisiológicos, focalizándose en las sensaciones de impotencia, incapacidad y vulnerabilidad. Desde esta vivencia alterada, es congruente la espera de ayuda por parte de la figura de apego. La frustración de esa petición de ayuda, se retroalimenta creando una mayor impotencia y sensación de desvalimiento. Cuanto más se exagera la necesidad de apoyo, 
menos posibilidades tiene de ser atendida, acrecentando el patrón ansioso. Este tipo de apego puede dañar la salud y hace muy difíciles las relaciones interpersonales, ya que las necesidades imperiosas de cuidado y protección, agotan los recursos del cuidador. Esta situación aparece en la vejez y es fácilmente reconocible en mayores que se presentan como desvalidos, exigentes, exagerados en sus reacciones y en su percepción de peligro, absolutamente enfocados en sus reacciones fisiológicas de temor, que interpretan como enfermedad, sin escuchar, ni encontrar a nadie que pueda cuidarlos con eficiencia, ya que sus demandas irracionales parecen no tener medida y situarse facilmente fuera de la realidad. El anciano con un patrón de apego ansioso tendría que ser reconocido, para poder comprender sus reacciones y para poder implmentar un sistema de reparación, en vez de una dejación del apoyo que solamente cronifica el sistema, creando un sufrimiento cada vez mayor que aumenta la sensacion subjetiva de vulnerabilidad y abandono, perjudicando gravemente la salud.

En el apego evitativo, no ha existido de forma consistente una figura disponible para la creación de seguridad básica, por lo cual la estrategia del sujeto es el bloqueo o la interrupción de las emociones. El objetivo no consciente, sería el de mantener las necesidades afectivas desconectadas, sobre todo la ansiedad, la tristeza, la culpa, la vergüenza, la ira, o los sentimientos de vulnerabilidad. También aparece una desconexión con la percepción de amenaza (Ein-Dor et al., 2010), porque la amenaza haría reactivar la necesidad de figuras de apego. Se trata de activar la exclusión defensiva, y suprimir todo componente emocional con objetivos de autoprotección (Milkunicer y Shaver, 2020). De este modo se reduce el sufrimiento, pero también la capacidad de admitir e integrar esas emociones, ocasionando lo que Bowlby definia como, aparición de síntomas mentales segregados (Bowlby, 1983). Observamos esta estrategia en mayores aparentemente tranquilos en situaciones objetivamente amenazantes, que no parecen necesitar nada, con percepción de que son autónomos en situaciones insostenibles donde verdaderamente no lo son. Permanecen aislados por propia convicción, aunque esto ocasione riesgo para su supervivencia. Sostienen con aparente tranquilidad actuaciones irracionales de falta de cuidado o protección, que ponen en riesgo su vida, porque no parecen valorar las amenazas de forma realista.

Las estrategias creadas por los apegos inseguros son adaptativas en principio, pues protegen y evitan el sufrimiento, ya que consiguen adaptarse a la convivencia con una figura de apego incoherente o no disponible. Pero estas estrategias se vuelven desadaptativas, porque privan al sujeto de la búsqueda de apoyo real y de la dependencia saludable de los otros, promoviendo inadecuación, desequilibrio emocional y enfermedad.

Las personas con apego seguro, son capaces de reconocer las situaciones amenazantes y luchar de manera constructiva, adoptando estrategias centradas en problemas y solicitando apoyo social. Frente a estas, las evitativas tienden a ignorar las dificultades distanciándose y sin contar con el apoyo social, y los tipos ansiosos, se vuelven pasivos y se enredan en componentes emocionales poco adaptativos para la resolución de problemas. Todo esto influye en las estrategias de afrontamiento, y se convierte en un componente importante de la regulación emocional. Las personas evitativas confian en el distanciamiento cognitivo y en la desconexión emocional, mientras que los apegos ansiosos se focalizan en sus propias señales de angustia, y valoran de forma desproporcionada las amenazas (Frías et al., 2014).

La presencia continua del apego, hace que este constructo tenga un lugar importante en la regulación emocional. La teoria del apego se considera una teoría de regulación emocional, definida como la capacidad para modular la respuesta fisiológica y la implementación de estrategias con la finalidad de ofrecer una respuesta ajustada al contexto (Thompson, 1994). Pero a diferencia de otras, pone el énfasis en los aspectos automáticos y en la presencia de diversas tipologías que explican las distintas respuestas emocionales. Asimismo, las características de aparición temprana y su tendencia a la estabilidad a lo largo del ciclo vital, hacen del apego, una especie de perequisito o condición previa, sobre la que se desarrollarían posteriormente todas las demás adquisiciones relativas a la regulación emocional. La respuesta de apego está en el origen del desarrollo evolutivo, y se impone de forma característica durante todo el ciclo vital y en la vejez, porque esta sujeta a automatismo, hunde sus raíces en la psicofisiologia y en 
las experiencias de infancia, y muestra tendencia a la estabilidad a través del ciclo vital. Pensar que en la vejez esta respuesta va a desaparecer sería un contrasentido, más bien explica muchas de las experiencias y reacciones características de los mayores, ofreciendo un marco comprensivo desde el cual es mas fácil entender y aliviar las innumerables dificultades emocionales que los apegos inseguros pueden provocar.

\section{Apego y emoción. Alcance de la influencia del apego en la vivencia emocional en la vejez}

Como hemos visto, la teoría del apego por su importancia en la regulación emocional y en la vivencia de las experiencias interpersonales, tendría un efecto directo en el estado de ánimo de las personas que envejecen (Shaver y Mikulincer, 2004). Observamos que los diferentes tipos de apego en la vejez, condicionan las diferentes vivencias emocionales.

Las personas que puntuan alto en apego ansioso o evitativo, parecen tener serias dificulades para identificar y describir emociones, así como para mantener la atención y los pensamientos en el presente. Encontraron deficits en la estructura neuronal de las regiones cerebrales implicadas en la regulación emocional, lóbulo temporal anterior, compatibles con un modelo neurotóxico de experiencias de apego ansioso que afecta tanto a la sensación de seguridad, como a la regulación de las emociones. También existen diferencias en expresión emocional y vivencia emocional, encontrando que los tipos inseguros sufren de mayor ansiedad y se enfadan más frecuentemente. En cuanto a la capacidad para acceder y manejarse con los recuerdos tristes o difíciles, los tipos ansiosos acceden más facilmente a los recuerdos negativos, pero posteriormente les resulta difícil inhibir estas emociones que se propagan y extienden fácilmente a otros aspectos de la realidad con gran intensidad. Los apegos evitativos sufrían de altos niveles de ansiedad defensiva y baja accesibilidad a los recuerdos negativos. El apego evitativo se asoció con una mayor capacidad para suprimir los pensamientos relacionados con la separación, con pensamientos de pérdida menos frecuentes después de la tarea de supresión, y una me- nor conductividad de la piel (Consedine et al., 2012; Pan, 2009).

El tipo evitativo, aparentemente autosuficiente, soporta una importante debilidad en sus defensas cuando las demandas emocionales se acumulan, con una disminución de la variabilidad de la frecuencia cardíaca, deterioro de la recuperación de la presión arterial, mayor conductividad de la piel y aumento de la presión arterial diastólica, en respuesta a factores estresantes de laboratorio. El aumento del apego evitativo en la vejez, no es en absoluto positivo, pues los apegos evitativos ante demandas crónicas y estresantes colapsan, causando niveles más altos de ansiedad que los apegos ansiosos, y una alta reactividad psicofisiológica que eleva el riesgo cardiovascular (Cassidy y Shaver, 2016). En tipos evitativos, la alerta psicofisiológica se mantiene continuamente, presentando una alta reactividad psicofisiológica, mientras que en ansiosos solamente se activa en el momento de la amenaza (Kim, 2006). De manera similar, las personas con patrones evitativos pueden manifestar fuertes emociones negativas y pérdida de autocontrol, en respuesta a eventos crónicos, incontrolables y gravemente angustiantes, en lo que podemos definir como una fractura de las defensas.

Sheinbaum et al. (2015) encontraron que los mayores con apego ansioso experiementaban mayor afecto negativo, estrés, y rechazo social percibido, mientras que los evitativos exhibían tendencias desactivadoras, como la disminución de emociones negativas y un menor deseo de estar con los demás. En una muestra de mayores, el apego seguro se relacionó con menos culpa, vergüenza, sensación de ofensa y con mayor alegría, tristeza, interés, miedo e irritación. El apego evitativo a su vez con una mayor alegría, prevención, vergüenza y ansiedad, y el ansioso correlacionaba con mayor interés y con menos alegría, vergüenza y miedo (Consedine y Magai, 2003). En un estudio diseñado para evaluar emociones, estilos de crianza y apego en adultos mayores, se encontraron expresiones faciales de alegría, falta de afecto negativo en apegos seguros; vergüenza y ansiedad en los evitativos y fantasias de cercanía, ira o depresión en los apegos ansiosos (Magai et al., 2000). 


\section{Apego y bienestar en la vejez}

Del mismo modo que los apegos inseguros producen problemas de salud y de malestar emocional, el apego seguro se muestra como uno de los elementos más característicos del bienestar, pudiendo considerarse en la vejez un importante recurso de resiliencia y de ajuste social. Es fundamental reseñar el influjo de los mecanismos del apego en la salud psicológica, en la percepción de salud física, en la adaptación a la enfermedad crónica, en la carga del cuidador, en las reacciones de duelo, en la vivencia religiosa, en el afrontamiento de la muerte y en la apertura a la vida (Bradley, 2001). En todos estos ámbitos, la teoría del apego realiza interesantes aportaciones, convirtiéndose en una perspectiva moduladora y novedosa para la comprensión de la vejez y del proceso de envejecimiento. Milkunicer y Shaver (2016) encontraron que las personas con apego seguro eran más optimistas hacía la vida, realizaban evaluaciones menos catástróficas de las amenazas, demostraban más confianza para enfrentar peligros y una utilización de estrategias de regulacion emocional más eficientes y constructivas (búsqueda de apoyos, resolución de problemas, reevaluacion). Además, mostraban un mejor manejo de los eventos amenazantes, o si estos eran inevitables, una reevaluacion en términos benignos y constructivos, permaneciendo en todo este proceso abiertos a la expresión de sus emociones, a comunicar sus sentimientos con libertad y a experimentarlos sin ser distorsionados.

El estilo de apego es una variable importante para la promoción de un envejecimiento saludable (Spence et al., 2018). Los resultados sostienen que los apegos inseguros están inversamente relacionados con la autoaceptación, el crecimiento personal, la calidad de las relaciones, el propósito en la vida y el manejo del ambiente. El apego seguro, sin embargo, facilita una actitud de armonía y aceptación de uno mismo (Homan y Sirois, 2017).

Desde el punto de vista de la intervención, aunque el apego seguro, se considera el estilo óptimo de apego, el objetivo de las intervenciones de apego en la vejez, no estaría en el intento de cambio de la tipología de apego. El objetivo sería mas bien, conseguir que los sujetos puedan funcionar con la menor angustia emocional posible (Ai y Zhang, 2012), y para ello habría que ajustar los comporta- mientos de cuidado al tipo de apego que presenta cada persona mayor, para así ayudarle a una correcta regulación emocional. Esta búsqueda se refiere al viejo concepto de bondad de ajuste, que, aplicado a la vejez, significaría adaptación y respeto al tipo de apego, ya este tiene características de patrón estructural estable que se activa de forma automática y no consciente.

\section{Evolución de los tipos de apego en la vejez y apegos simbólicos}

Es difícil en la actualidad realizar afirmaciones sobre la estabilidad del apego por las razones de las dificultades en la evaluación del constructo, y también por una falta de diseños longitudinales a lo largo del ciclo vital (Scharfe y Bartholomew, 1994). Los apegos inseguros, como hemos visto, producen tantos síntomas y dificultades durante el envejecimiento, que es importante saber que cantidad de mayores podrían estar siendo una población de riesgo.

La mayor parte de los trabajos sugieren estabilidad en los patrones de apego hasta la edad adulta, aunque hemos de contar con un $30 \%$ de variación, o con variaciones mayores, si estamos ante situaciones traumáticas o desestabilizadoras sobre una tendencia general hacia la estabilidad. Ante la falta de estudios longitudinales, una aproximación a estos datos sería comparar los resulados obtenidos en muestras de edad adulta intermedia y en muestras de mayores. En esta comparación nos encontraríamos que en la edad adulta intermedia o edad juvenil, encontramos un apego ansioso entre 5 y $9 \%$, un apego evitativo, entre 16 y $30 \%$, y un apego seguro entre 52 y $65 \%$ (BakermansKranenburg y Van IJzendoorn, 2009; Cassidy y Shaver, 1999; Feeney y Noller, 1990; Guzman y Contreras, 2012; Hazan y Shaver, 1987; Magai et al., 2016; Roissman, 2007).

Frente a estos resultados, los estudios en mayores indican un aumento de los patrones evitativos, que pasan a suponer entre el 37 y el $52 \%$ de la población (Diehl et al., 1998; Magai et al., 2000; Webster, 1997). Así lo señalan los diversos autores como Van Asche et al. (2013), junto con 1 aexistencia de una significativa disminución del apego ansioso (Mickelson et al., 1997). 
Además de revisar el tipo de apego y su continuidad en el tiempo, es interesante analizar si las figuras de apego cambian en esta época de la vida. En la vejez se produce con frecuencia la pérdida de figuras de apego, apareciendo entonces un cambio en la naturaleza y en las identidades de dichas las figuras. Surge el apego a los seres queridos fallecidos y a Dios, son los llamados apegos simbólicos. Los apegos simbólicos, satisfacen las necesidades de vinculación emocional y suponen una fuente de comodidad y seguridad a través de los recuerdos, ya que la representación mental de las figuras de apego, facilita la regulación del afecto, reduciendo los pensamientos negativos y facilitando la recuperación frente a recuerdos perturbadores y factores estresantes externos (Selcuk et al., 2012). El segundo tipo de apego simbólico, es el apego a Dios (Granqvist et al., 2010). La religión proporciona figuras de apego sustitutas, que regulan la angustia y promueven sentimientos de seguridad, además de una disminución del dolor percibido (Hatefi et al., 2019). Homan (2014) encuentra que el apego seguro a Dios, predice unas relaciones positivas con los demás, autoaceptación, manejo adecuado del ambiente y sensación de crecimiento personal.

\section{Conclusiones}

El apego es una respuesta autómatica de inicio muy temprano, que continua presente durante todo el ciclo vital activándose cuando se percibe alguna situación amenazante. En la vejez estas situaciones aumentan, activandose por lo tanto con más frecuencia las respuestas de apego, que de ser desadaptativa, estaría ocasionando dificultades añadidas que es imprescindible tener en cuenta.

El apego es un factor previo que tendríamos que contemplar en cualquier estudio o evaluación de la regulación emocional del anciano, por sus características de activación automática y por su conexión con mecanismos biológicos básicos de regulación de la ansiedad. Los apegos inseguros actúan en la vejez produciendo respuestas de extrema dependencia y sufrimiento en los apegos ansiosos, y también de independencia excesiva, que impide el autocuidado y la conciencia de vulnerabilidad, en los tipos evitativos. Además, los problemas de salud, propios de la vejez, se agravan en los sujets con tipologías de apego in- seguro, creando una mayor vulnerabilidad a la enfermedad por los procesos biológicos subyacentes de tipo inflamatorio y cardiaco que están asociados a las respuestas de apego.

La proporción de personas en riesgo por la presencia de apegos inseguros, es más de la mitad de la población de los mayores. Comprender mejor las reacciones de las personas mayores sometidas a tensión e intervenir de un modo ajustado, es el camino que se abre a través de la teoria del apego, pues esta perspectiva se convierte en moduladora y novedosa para la comprensión de la vejez y del proceso de envejecimiento.

\section{Referencias}

Ai J. y Zhang M. (2012). Study on the Relationship between the Elderly Loneliness, Suicide Attitude and Attachment Style. Chinese Health Psychology Journal of China Psychological Health, 20(6), 830-833.

Bakermans-Kranenburg, M. J. y van IJzendoorn, M. H. (2009). The First 10,000 Adult Attachment Interviews: Distributions of adult attachment representations in clinical and non-clinical groups. Attachment \& Human Development, 11(3), 223263. https://doi.org/10.1080/14616730902814762

Bowlby, J. (1983). La pérdida afectiva. Paidós.

Bradley, J. M. y Cafferty, T. P. (2001). Attachment among older adults: Current issues and directions for future research. Attachment \& Human Development, $\quad 3(2)$, 200-221. https://doi.org/10.1080/14616730110058016

Cassidy, J. y Shaver, P. R. (2016). Handbook of attachment: Theory, Research, and Clinical Applications ( $3^{\mathrm{a}}$ ed.). The Guilford Press.

Chen, E., Miller, G. E., Kobor, M. S. y Cole, S. W. (2011). Maternal Warmth Buffers the Effects of Low Early-Life Socioeconomic Status on pro- 
Inflammatory Signaling In Adulthood. Molecular Psychiatry, 16(7), 729-737. https://doi.org/10.1038/mp.2010.53

Chung, H. Y., Cesari, M., Anton, S., Marzetti, E., Giovannini, S., Seo, A. Y., Carter, C, Yu, B. P. y Leeuwenburgh, C. (2009). Molecular Inflammation: Underpinnings of Aging and Agerelated Diseases. Ageing Research Reviews, 8(1), 18-30. https://doi.org/10.1016/j.arr.2008.07.002

Coan, J. A. (2008). Toward a neuroscience of attachment. En J. Cassidy y P. R. Shaver (Eds.), Handbook of Attachment: Theory, Research, and Clinical Applications (2 ${ }^{\mathrm{a}}$ ed.; pp. 241-265). The Guilford Press.

Consedine, N. S. y Magai, C. (2003). Attachment and Emotion Experience in Later Life: The View from emotions theory. Attachment \& Human Development, 5(2), 165-187.

Consedine, N. S., Fiori, K. L. y Magai, C. (2012). Regulating Emotion Expression and regulating Emotion Experience: Divergent Associations with Dimensions of Attachment Among Older Women. Attachment \& Human Development, 14(5), 477500. https://doi.org/10.1080/14616734.2012.706433

Diehl, M., Elnick, A. B., Bourbeau, L. S. y LabouvieVief, G. (1998). Adult Attachment Styles: Their Relations to Family Context and Personality. Journal of Personality and Social Psychology, 74(6), 1656-1669. https://doi.org/10.1037/0022$\underline{3514.74 .6 .1656}$

Ein-Dor, T., Mikulincer, M., Doron, G. y Shaver, P. R. (2010). The Attachment Paradox: How Can so many of us (the insecure ones) Have no Adaptive Advantages? Perspectives on Psychological Science, 5(2), 123-141. https://doi.org/10.1177/1745691610362349

Ein-Dor, T., Verbeke, W. J. M. I., Mokry, M. y Vrtička, P. (2018). Epigenetic Modification of the Oxytocin and Glucocorticoid Receptor Genes is Linked to Attachment Avoidance in Young Adults. Attachment \& Human Development, 20(4), 439454.

https://doi.org/10.1080/14616734.2018.1446451

Feeney, J. A. y Noller, P. (1990). Attachment Style as a Predictor of Adult Romantic Relationships. Journal of Personality and Social Psychology, 58(2), 281291. https://doi.org/10.1037/0022-3514.58.2.281

Frías, M. T., Shaver, P. R. y Díaz-Loving, R. (2014). Individualism and Collectivism as Moderators of the Association between Attachment Insecurities, Coping, and Social Support. Journal of Social and Personal Relationships, 31(1), 3-31.

Gouin, J. P., Glaser, R., Loving, T. J., Malarkey, W. B., Stowell, J., Houts, C. y Kiecolt-Glaser, J. K. (2009). Attachment Avoidance Predicts Inflammatory Responses to Marital Conflict. Brain, Behavior, and Immunity, 23(7), 898-904. https://doi.org/10.1016/j.bbi.2008.09.016

Granqvist, P., Mikulincer, M. y Shaver, P. R. (2010). Religion as attachment: Normative processes and individual differences. Personality and Social Psychology Review, 14(1), 49-59. https://doi.org/10.1177/1088868309348618

Guzmán, M. y Contreras, P. (2012). Estilos de apego en relaciones de pareja y su asociación con la satisfacción marital [Attachment Styles in Couples and their Association with Marital Satisfaction]. Psykhe: Revista de La Escuela de Psicología, 21(1), 69-82. https://doi.org/10.4067/S0718$\underline{22282012000100005}$

Hatefi, M., Tarjoman, A. y Borji, M. (2019). Do Religious Coping and Attachment to God Affect Perceived Pain? Study of the Elderly with Chronic Back Pain in Iran. Journal of Religion and Health, 58(2), 465-475. https://doi.org/10.1007/s10943018-00756-9 
Hazan, C. y Shaver, P. (1987). Romantic Love conceptualized as an Attachment Process. Journal of Personality and Social Psychology, 52(3), 511524. https://doi.org/10.1037/0022-3514.52.3.511

Hofer, M. A. (2006). Psychobiological Roots of Early Attachment. Current Directions in Psychological Science, 15(2), 84-88. https://doi.org/10.1111/j.0963-7214.2006.00412.x

Homan, K. J. (2014). Symbolic Attachment Security and Eudemonic Well-Being in Older Adults. Journal of Adult Development, 21(2), 89-95. https://doi.org/10.1007/s10804-013-9182-6

Homan, K. J. y Sirois, F. M. (2017). Self-Compassion and Physical Health: Exploring the Roles of Perceived Stress and Health-Promoting Behaviors. Health Psychology Open, 4(2), 1-9. https://doi.org/10.1177/2055102917729542

Iyengar, U., Rajhans, P., Fonagy, P., Strathearn, L. y Kim, S. (2019). Unresolved Trauma and Reorganization in Mothers: Attachment and Neuroscience Perspectives. Frontiers in Psychology, 10. https://doi.org/10.3389/fpsyg.2019.00110

Jaremka, L. M., Glaser, R., Loving, T. J., Malarkey, W. B., Stowell, J. R. y Kiecolt-Glaser, J. K. (2013). Attachment Anxiety is Linked to Alterations in Cortisol Production and Cellular Immunity. Psychological Science, 24(3), 272-279. https://doi.org/10.1177/0956797612452571

Ketay, S. y Beck, L. A. (2017). Attachment Predicts Cortisol Response and Closeness in Dyadic Social Interaction. Psychoneuroendocrinology, 80, 114121.

https://doi.org/10.1016/j.psyneuen.2017.03.009

Kim, Y. (2006). Gender, Attachment, and Relationship Duration on Cardiovascular Reactivity to Stress in a Laboratory Study of Dating Couples. Personal Relationships, 13(1), 103-114. https://doi.org/10.1111/j.1475-6811.2006.00107.x
Long, M., Verbeke, W., Ein-Dor, T. y Vrtička, P. (2020). A Functional Neuro-Anatomical Model of Human Attachment (NAMA): Insights from first- and Second-Person Social Neuroscience. Cortex: $A$ Journal Devoted to the Study of the Nervous System and Behavior, 126, 281-321. https://doi.org/10.1016/j.cortex.2020.01.010

Magai, C., Hunziker, J., Mesias, W. y Culver, L. C. (2000). Adult Attachment Styles and Emotional Biases. International Journal of Behavioral Development, 24(3), 301-309. https://doi.org/10.1080/01650250050118286

Magai, C. (2016). Attachment in middle and later life. En J. Cassidy y P. R. Shaver (Eds.), Handbook of attachment: Theory, research, and clinical applications ( $2^{\mathrm{a}}$ ed. pp. 534-552). The Guilford Press.

McMahon, G., Creaven, A. y Gallagher, S. (2019). Perceived Social Support Mediates the Association between Attachment and Cardiovascular Reactivity in Young Adults. Psychophysiology. https://doi.org/10.1111/psyp.13496

Meaney, M. J. (2001). Maternal care, Gene Expression, and the Transmission of Individual Differences in Stress Reactivity Across Generations. Annual Review of Neuroscience, 24, 1161-1192. https://doi.org/10.1146/annurev.neuro.24.1.1161

Mickelson, K. D., Kessler, R. C. y Shaver, P. R. (1997). Adult Attachment in a Nationally Representative Sample. Journal of Personality and Social Psychology, 73(5), 1092-1106. https://doi.org/10.1037/0022-3514.73.5.1092

Mikulincer, M. y Shaver, P. R. (2016). Adult Attachment and Emotion Regulation. En J. Cassidy y P. R. Shaver (Eds.), Handbook of Attachment: Theory, Research, and Clinical Applications ( $3^{\mathrm{a}}$ ed.; pp. 507-533). The Guilford Press.

Mikulincer, M. y Shaver, P. R. (2019). Attachment Orientations and Emotion Regulation. Current 
Opinion in Psychology, 25, 6-10. https://doi.org/10.1016/j.copsyc.2018.02.006

Mikulincer, M. y Shaver, P. R. (2020). Broaden-andbuild Effects of Contextually Boosting the Sense of Attachment Security in Adulthood. Current Directions in Psychological Science, 29(1), 22-26. https://doi.org/10.1177/0963721419885997

Miller, G. E., Chen, E. y Parker, K. J. (2011). Psychological Stress in Childhood and Susceptibility to the Chronic Diseases of Aging: Moving toward a Model of Behavioral and Biological Mechanisms. Psychological Bulletin, 137(6),

959-997. https://doi.org/10.1037/a0024768

Pan, J. (2009). Different Attachment Styles, Social Support and Subjective Sense of Happiness of the Elderly. Jiangxi Normal University.

Picardi, A., Battisti, F., Tarsitani, L., Baldassari, M., Copertaro, A., Mocchegiani, E. y Biondi, M. (2007). Attachment Security and Immunity in Healthy Women. Psychosomatic Medicine, 69(1), 40-46.

https://doi.org/10.1097/PSY.0b013e31802dd777

Pietromonaco, P. R., DeBuse, C. J. y Powers, S. I. (2013). Does Attachment Get under the Skin? Adult Romantic Attachment and Cortisol Responses to Stress. Current Directions in Psychological Science, 22(1), 63-68. https://doi.org/10.1177/0963721412463229

Quiroga Méndez M. P. (2018). Apego y Vejez, aportaciones al desarrollo evolutivo. En Actas del $V$ Congreso Internacional de Investigación en Salud y Envejecimiento. Vol 1.

Roisman, G. I., Fraley, R. C. y Belsky, J. (2007). A Taxometric Study of the Adult Attachment Interview. Developmental Psychology, 43(3), 675686. https://doi.org/10.1037/0012-1649.43.3.675
Scharfe, E. y Bartholomew, K. (1994). Reliability and Stability of Adult Attachment Patterns. Personal Relationships, $1(1)$,
https://doi.org/10.1111/j.1475$\underline{6811.1994 . t b 00053 . x}$

Selcuk, E., Zayas, V., Günaydin, G., Hazan, C. y Kross, E. (2012). Mental Representations of Attachment Figures Facilitate Recovery following Upsetting Autobiographical Memory Recall. Journal of Personality and Social Psychology, 103(2), 362378. https://doi.org/10.1037/a0028125

Shaver, P. R. y Mikulincer, M. (2004). Attachment in the Later Years: A Commentary. Attachment \& Human Development, 6(4), 451-464. https://doi.org/10.1080/1461673042000303082

Sheinbaum, T., Kwapil, T. R., Ballespí, S., Mitjavila, M., Chun, C. A., Silvia, P. J. y Barrantes-Vidal, N. (2015). Attachment Style Predicts Affect, Cognitive Appraisals, and Social Functioning in Daily Life. Frontiers in Psychology, 6. https://doi.org/10.3389/fpsyg.2015.00296

Shirtcliff, E. A., Skinner, M. L., Obasi, E. M. y Haggerty, K. P. (2017). Positive Parenting Predicts Cortisol Functioning six Years Later in Young Adults. Developmental Science, 20(6). https://doi.org/10.1111/desc.12461

Sippel, L. M., Han, S., Watkins, L. E., Harpaz-Rotem, I., Southwick, S. M., Krystal, J. H., Olff, M., Sherva, R., Farrer, L. A., Kranzler, H. R., Gelernter, J. y Pietrzak, R. H. (2017). Oxytocin Receptor Gene Polymorphisms, Attachment, and PTSD: Results from the National Health and Resilience in Veterans Study. Journal of Psychiatric Research, 94, 139-147. https://doi.org/10.1016/j.jpsychires.2017.07.008

Spence, R., Jacobs, C. y Bifulco, A. (2018). Attachment Style, Loneliness and Depression in Older Age Women. Aging \& Mental Health, 24(5), 837-839. https://doi.org/10.1080/13607863.2018.1553141 
Shunqin, Y. (2015). Review of Effects of "attachment" of the elderly on physical and mental health of old chronic patients. Studies in Asian Social Science, 2(2), 15. https://doi.org/10.5430/sass.v2n2p15

Thompson, R. A. (1994). Emotion regulation: A theme in search of definition. Monographs of the Society for Research in Child Development, 59(2-3), 2552. https://doi.org/10.2307/1166137

Van Assche, L., Luyten, P., Bruffaerts, R., Persoons, P., van de Ven, L. y Vandenbulcke, M. (2013). Attachment in old age: Theoretical assumptions, empirical findings and implications for clinical practice. Clinical Psychology Review, 33(1), 6781. https://doi.org/10.1016/j.cpr.2012.10.003

Vrtička, P., \& Vuilleumier, P. (2012). Neuroscience of Human Social Interactions and Adult Attachment Style. Frontiers in Human Neuroscience, 6. https://doi.org/10.3389/fnhum.2012.00212

Webster, J. D. (1997). Attachment Style and Well-Being in Elderly Adults: A Preliminary Investigation. Canadian Journal on Aging, 16(1), 101-111. https://doi.org/10.1017/S0714980800014185 
